\title{
Network pharmacology and bioinformatics approach reveals the therapeutic mechanism of action of curcumin in Alzheimer disease
}

\section{Deepanshi Vijh}

Himachal Pradesh University

Md. Ali Imam

Jamia Millia Islamia

Mohd Maksuf UI Haque

Jamia Millia Islamia Central University: Jamia Millia Islamia

Subhajit Das

NCCS: National Centre For Cell Science

Asimul Islam

Jamia Millia Islamia

Md Zubbair Malik ( $\nabla$ zubbairmalik@jnu.ac.in )

Jawaharlal Nehru University https://orcid.org/0000-0001-6693-0401

\section{Research Article}

Keywords: Alzheimer disease, curcumin, molecular docking, network pharmacology

Posted Date: February 2nd, 2022

DOI: https://doi.org/10.21203/rs.3.rs-1287163/v1

License: (1) This work is licensed under a Creative Commons Attribution 4.0 International License.

Read Full License 


\section{Abstract}

Curcumin possesses antioxidant and anti-inflammatory properties and shows a major role in reducing the amyloid plaques formation which is the major cause of Alzheimer's disease (AD). Consequently, a methodical approach was used to select the potential protein targets of curcumin in AD through network pharmacology. In this study, through integrative methods $A D$ targets of curcumin through SwissTargetPrediction databases, STITCH database, BindingDB, PharmMapper, Therapeutic Target Database (TTD), Online Mendelian Inheritance in Man (OMIM) database were predicted followed by gene enrichment, network construction, network topology, and docking studies. Gene ontology analysis facilitated identification of a list of possible $A D$ targets of curcumin (74 targets genes). The correlation of the obtained targets with AD was analysed by Gene Ontology (GO) pathway enrichment analyses and Kyoto Encyclopaedia of Genes and Genomes (KEGG). Using network pharmacological approach, we identified five key genes (RARA, APP, PRARG, STAT3 and MAPK1). Further, we performed molecular docking for analysing the mechanism of curcumin. To validate the temporospatial expression of key genes in human central nervous system (CNS), we searched the Human Brain Transcriptome (HBT) dataset. This study concluded with novel insights into the therapeutic mechanisms of curcumin which in the future will ease the treatment of $A D$ with the clinical application of curcumin.

\section{Introduction}

Alzheimer's is an age-linked neurological disorder that results in progressive and irreversible cognitive impairment. It is characterized by memory loss, psychosis, and reduction in the motor skills of the affected individual caused because of the shrinkage of the brain and irreversible damage to the brain cells and their connections. The disease is prevalent in middle-aged and older people especially in the United States with 5.8 million of the affected population which is predicted to reach the 13.8 million marks by 2050 [1].The key attributes of the disease are deposits of beta-amyloid proteins called plaques and the tangles of fibres of a protein called tau [2]. These abnormal structures are responsible for the damage and loss of connection between the nerve cells. Many pharmacological and nonpharmacological approaches have been studied to improve the symptoms and mitigate the pathological changes of AD. Some of the approved drugs available for the treatment are N-methyl D-aspartate (NMDA) receptor blockers i.e. memantine, Acetyl Cholinesterase inhibitors (donepezil, rivastigmine, and galantamine, [3]. All these available therapies and drugs are designed by targeting these abnormal protein structures or are based on the chlorogenic hypothesis and they only target the symptoms but not the pathogenesis, therefore, there is no cure for the disease developed despite the many ongoing research and clinical trials [4]. Therefore, new treatments are needed.

Ayurvedic medicine is one of the traditional systems of medicine indigenous to India and has gained attention as they are multi-targeted and have fewer side effects. Ayurvedic system of medicine describes the therapeutic properties of many indigenous plants and their application for the treatment of many neurological disorders, like dementia. Due to the side effects of current pharmacological treatment, recently systematic research has been conducted, to understand the results of these herbs on brain 
disorders such as AD [5] [6]. Many herbal plants are used in various Ayurvedic medicines like Acorus calamus, Bacopa monniera, Ginkgo biloba, Epimedium koreanum, Curcuma Longa, etc actas memory boosters and are shown to have anticholinesterase activity [6]. Curcumin (diferuloylmethane) is a polyphenolic compound found in a commonly used Indian spice called turmeric [7]. Because of its antiinflammatory, antioxidant, and cancer chemo-preventive properties, it is shown to diminish oxidative damage and amyloid plaques formation [8] [9]. Network pharmacology has emerged as a promising area and a new approach to advanced drug discovery and comprehending the relationship between diseases and drugs at a systematic level [10].To elucidate the possible course of action of curcumin as the medicament of $A D$. This analysis used the technique of network pharmacology to decipher the targets protein linked to curcumin against $A D$.

\section{Materials And Methods}

Through the process of target mining and the use of many online tools the targets of curcumin and the genes related to Alzheimer's were identified. The potential anti-AD genes were selected by the intersection of these groups. Protein-protein interaction (PPI) network, Varelect database, KEGG pathway and Gene Ontology (GO) was analyzed for the potential targets. The key genes obtained from the above analysis were further detected for their expression in different brain regions. At last molecular docking was performed on the key genes observed in the PPI analysis. The work scheme is shown in Fig. 1.

\section{Analysis of drug-likeness}

On the basis of Lipinski's rule of five (RO5) drug-likenesses of the possible therapeutic compound was observed on the basis of few parameters. These properties contain topological polar surface area (TPSA), molecular weight (MW), XLogP3 (water-octanol partition coefficient), hydrogen bond donor count, number of rotatable bonds, and hydrogen bond acceptor count. The other important factors of pharmacokinetics which are important in the process of drug discovery are the brain access, bioavailability score, and gastrointestinal absorption. Bioavailability score depends on number of factors but it depends mostly on the gastrointestinal absorption [11] [12]. Brain Or IntestinaL EstimateD Prediction (BOILED-Egg) method is used for accurate prediction of this $\mathrm{Gl}$ absorption based on the polar and lipophilic character of the small molecule [12]. Curcumin's SMILES format

$(C O C 1=C(C=C C(=C 1) C=C C(=0) C C(=0) C=C C 2=C C(=C(C=C 2) 0) O C) 0$ was downloaded from the PubChem database (pubchem.ncbi.nlm.nih.gov) and uploaded into the Swiss-ADME server

(http://www.swissadme.ch/), a web-based tool to access the bioavailability, Gastrointestinal absorption and drug-likeness of the small molecules [11] [13].

\section{Predicting targets of curcumin}

The Potential Targets were collected from SwissTargetPrediction databases (http://www.swisstargetprediction.ch/) server [11], STITCH database(http://stitch.embl.de/), BindingDB (https://www.bindingdb.org/bind/index.jsp) [14] and The PharmMapper (http:// lilab.ecust.edu.cn/pharmmapper/) server [15]. The predicted targets were obtained by using the SMILES 
format of curcumin in BindingDB and Swiss Target Prediction database. This was based on the principles of structural similarity. The compound's name (Curcumin) was used to search the targets in STITCH database [16]. Moreover, curcumin's structure file (PubChem CID: 969561) was imported into PharmMapper server for downloading possible targets by applying the pharmacophore mapping method. Then, the derived targets were normalized using UniProt Database (http://www.uniprot.org/). Next, the potential target proteins derived from various sources were integrated and redundant targets were eliminated. Finally, 376 targets genes were collected from different databases after merging and removing redundant genes. This set of genes was called "targets of curcumin".

\section{Collecting targets related to Alzheimer's disease}

To certify the complete collection of the disease-associated genes, AD-related targets were redeemed from Therapeutic Target Database (TTD, http://bidd.nus.edu.sg/group/cjttd/), Comparative Toxicogenomics Database (http://ctdbase.org/), and Online Mendelian Inheritance in Man (OMIM) database (https://www.omim.org/), and CooLGeN database (http://ci.smu.edu.cn/CooLGeN/). In the source database used above phenotype keyword Alzheimer's was set. The targets proteins having hit scores more than 5 were identified as AD-associated genes from the CooLGen database [17]. The set of gene called "genes of $A D$ " containing 495 genes was finalised after merging the collected genes and removing redundant data. The convergence of the above two sets of targets proteins can be possible anti-AD targets of curcumin.

\section{Phenotypic- genotypic correlation analysis}

The VarElect is a free-online phenotype dependent database that allows for effective screening and rapid prioritization direct and indirect association between genes and diseases [18] [19]. The online VarElect tool was used for the correlational analysis between the "anti-AD targets" and the phenotype of "AD". A main role of VarElect analysis tool is its ability to perform gene list elucidation and scoring done on the basis of phenotypic keywords entered by the user [20]. The scoring method used in VarElect derived basically from Elastic search technology [21]. The score of a specific phenotype is determined by its recurrence in the individual Genecards database, in comparison to that in all genes [20].

\section{Protein-Protein Interaction}

The Protein-Protein Interaction (PPIs) are substantial for the comprehension of individual protein function and complex cellular pathways that associated in the development of diseases [22]. In accordance to the latest studies PPIs are related to many diseases like cancer, Alzheimer's and other neurodegenerative diseases and have potential to act as a promising strategy for the drug design and artificial designing of proteins [23]. Protein-protein interaction network mapping was done on common genes of Curcumin and $A D$, and the genes related with them were analysed using GeneMANIA database (https://genemania.org/) with the species limited to "Homosapiens". The PPI network was built using Cytoscape (version 3.6.1) which is employed for the integration and visualization of the data [24]. Network topological properties 
like the degree, betweenness and closeness centrality was analysed making use of network Analyzer a plugin of Cytoscape.

\section{Topological properties of network}

Topological analysis helps to understand a network's structure, which makes it easy to understand the hidden mechanisms [25]. The centrality measurement of the PPI network of AD associated genes are defined by betweenness (CB), and closeness (Cc). Using Network Analyzer [26] centrality measurements were calculated. These topological parameters were used to monitor the topological changes that occurred with network perturbation. In order to investigate the essential behaviours of the network, the following network properties were analysed.

\section{Degree}

During the network analysis, the total number of links established by a node in the network is called degree and indicated by $k$. In the process of network regulation, it is considered as measure of the local significance of a node. The graph is depicted by $G=(N, E)$, where $E$ denotes the edges while the nodes are denoted by $\mathrm{N}$.

Betweenness and closeness centrality are the basic parameters and centrality measures for the approximation of global functional significance of node in a network regulation [27].

\section{Betweenness centrality}

It is a measure of the extent of speed of communication of a node among other pairs of nodes in the network. This nodal measure is calculates as the number of shortest paths-path traffic from all possible routes passing through a node $u$ (Equation 1 ).

$$
c_{B}(u)=\sum_{v \in w \neq u} \frac{\sigma_{v w}(u)}{\sigma_{v w}} \quad \text { (Equation 1) }
$$

The betweenness centrality is the parameter of the capability of a node to extricate benefit from information flow all through the network[28]and its capability to control the signal processing over the other nodes through the network [27]

\section{Closeness centrality}

It measures closeness of a node from the other nodes in a network i.e., how short a path is. The nodal measure is calculated as the inverse of the sum of distances between the node of interest $u$ and all other $v \vee u$ (Equation 2).

$$
c_{C}(u)=\frac{1}{\sum_{v \neq u} d(u, v)}
$$


Closeness centrality depicts the speed of information distribution within a network i.e. from one node to other connected nodes [26]. The function of the node is depends upon the neighbour's centralities, and changes in accordance with the types of network association. There are very few chances that node remains isolated in such closely connected region of those nodes. Thus, it is a influential indicator of information conveyance source of a node within a network [29].

\section{Gene Ontology Enrichment Analysis}

The Gene Set Enrichment Analysis (GSEA) is a powerful analytical tool which is used for elucidating gene expression data which was generated by genome-wide experiments. These enrichment analyses tools help to connect previous knowledge to newly generated data and therefore discover the nature of genes in the conditions of health and disease [30] [31]. The Enrichr was used for the KEGG and GO analysis for the targets predicted against AD. Enrichr is an extensive web server for enrichment analysis and annotation of gene. It consolidates various databases like KEGG, GEO, GO, PPI databases, ChEA 2016, OMIM, etc [32]. Another comprehensive database called Metascape was used for enrichment analysis. It is a tool which is used to perform multi-platforms OMICs data interpretation and analysis [33]. To perform the enrichment analysis and gene annotation following steps were taken: Firstly, the curcumin related anti $A D$ gene list was inputted into the Enrichr and Metascape web server. Secondly the information about the signalling pathway was selected on the basis of the clinical and the pathological data.

\section{SynGO Gene Ontology}

On the basis of neurobiological and genetic evidence abnormally regulated synaptic genes are considered responsible for the aberrant brain plasticity which in turn is the convergent factor of brain disorders like Autism, Parkinson's and Alzheimer's disease [34]. SynGO is a global, publicly available database which is used for synapse research, analysis and elucidation of omics data on a large scale. The large number of genes present on this database which are highly enriched amidst the genes related to brain disorders. This database contains exclusive annotations based on expert-curated and published information [35]. The list of anti-AD genes was imputed into the SynGO online database and visualization tool to analyse and evaluate function and locations of synaptic genes to perform enrichment studies.

\section{Validation of spatiotemporal expression patterns of five key gene in various regions of human brain}

We identified the expression pattern of RARA, APP, PRARG, STAT3 and MAPK1 in various tissues of brain and developmental stages using BRAINEAC (www.braineac.org/). It is a large exon-specific eQTL database covering ten different regions of brain obtained from 134 post-mortem brains of the people belonging to European descent without any brain disorders. This database was used to inspect spatial dynamics of RARA, APP, PRARG, STAT3 and MAPK1 expression in all covered brain regions. BRAINEAC database to conduct e-QTL analysis. The data of this database was derived from MRC Sudden Death Brain Bank in Edinburgh, UK and the Sun Health Research Institute, which includes 10 regions of the brain 
(tem-poral cortex, hippocampus, medulla, putamen, occipital cortex, thalamus, intralobular white matter, substantia nigra, frontal cortex, and cerebellum)[36].

\section{Molecular Docking}

To evaluate the strength and the mode of interaction between the key proteins obtained and the curcumin molecule, molecular docking was performed. This helped to obtain deep understanding of the mechanism of action between the curcumin and the key target protein. The molecular docking was conducted by the Glide tool from Schrodinger maestro. Glide makes use of chain of hierarchical filters to find probable positions of the ligand in the active-site region of the receptor and provides superior robustness in the binding mode prediction [37]. The protein crystal structure was acquired by the Protein Data Bank database. Then the protein was prepared to remove the waters and any other associated molecules. The 3D structure of the ligand was downloaded in the SDF file from the PubChem Database. Finally, the ligand docking was done using Glide tool. The binding energy and the mode of interaction were studied after that.

\section{Results}

\section{Molecular Properties of Curcumin}

The Lipinski rule of five (RO5) establish pharmacokinetic properties of drugs such as distribution, metabolism, absorption, and excretion on specific molecular properties. In accordance with R05, the potential compound's molecular weight should be at most 500 Dalton, the rotatable bond below10, the hydrogen bond acceptors below10, and the hydrogen bond donors below 5, calculated (XLogP3-AA) octanol / water partition coefficient (XLogP3-AA) of no more than 5 and Polar surface area (PSA) no more than $140 \mathrm{~A}^{2}$. Our results indicated that our results were in accordance with R05, 0.55 Bioavailability score and High gastrointestinal absorption showing that curcumin has good drug-like properties without any violations (Table 1 ). The drugs with bioavailability score 0.55 or more have good pharmacokinetic properties and are considered potential candidates for oral drugs [38].

Table 1: Curcumin's Molecular Properties 


\begin{tabular}{|ll|}
\hline Property & Value \\
\hline Molecular Weight & $368.38 \mathrm{~g} / \mathrm{mol}$ \\
\hline XLogP3-AA & 3.20 \\
\hline PSA & $93.06 \AA^{2}$ \\
\hline Rotable Bonds & 8 \\
\hline H-bonds donor & 2 \\
\hline H-bonds acceptor & 6 \\
\hline Molar refractivity & 102.80 \\
\hline Bioavailability Score & 0.55 \\
\hline Gl Absorption & High \\
\hline Blood Brain Barrier Permeability & No \\
\hline
\end{tabular}

\section{Analysis between Targets of Curcumin and AD}

In this study, the targets of the curcumin were predicted using the STITCH, the Binding DB, the PharmMapper and SwissTargetPrediction databases. After merging target proteins obtained, 383 targets for curcumin were obtained. Additionally, AD-linked genes were retrieved from the OMIM, CTD, TTD, and CooLGeN databases. 512 targets were obtained after redundant data were deleted. Finally, 74 genes were selected from the intersection of the curcumin targets and AD targets. A drug exhibits characteristics called as polypharmacology or drug promiscuity which means it can bind to multiple targets. Thereby, it is important to understand the drug-target interactions. To study the relationship between curcumin and the targets, a compound-target network (C-T network) was built. This network had 75 nodes and 74 edges and is presented in Fig. 2.

\section{Gene enrichment analysis}

We conducted KEGG pathway and GO enrichment analyses to expound the potential biological functions of 74 target genes. GO annotation and KEGG pathway analyses for the 74 targeted genes were performed. The significant enrichment of these targeted genes are shown in Fig.3.The targeted gene were enriched in molecular function (MF) including transmembrane receptor protein kinase activity, transition of metal ion binding, MAP kinase activity etc (Fig. 3A). As for cellular component the targeted genes are involved in dendrite, neuron projection, axon, nucleus, intracellular organelle lumen etc (Fig. 3B). According to the $\mathrm{GO}$ enrichment results these targets not only regulate Protein phosphorylation, transferase activity, inflammatory response and peptidyl-tyrosine modification (Fig. 3A) but also regulate the Protein kinase binding, Protein homodimerization activity and DNA binding (Fig. 3C). As shown in these Fig. $\mathbf{3 C}$ top 10 terms in biological process and Molecular process were related to "protein 
phosphorylation" (GO: 0006468), regulation of "inflammatory response" (GO: 0050727) and regulation of "kinase activity" (GO: 0043549). All these are mainly related to development and spread of the AD.

Additionally, the pharmacological mechanism of the curcumin against the $A D$ was studied using the KEGG analysis using the Enrichr tool and DisGeNET analysis using the Metascape online database. After the enrichment analysis of the pathway 74 genes were mapped to 171 pathways. The results showed that many targets played role in multiple biological pathways simultaneously (Fig. 3E). For example, both KDR and MAPK1 were both involved in the Ras signalling pathway (Fig. 3D). It has been studies that the activation of the Ras-ERK signalling is responsible for the hyperphosphorylation of the tau proteins another major hallmark for the AD [39]. The results indicated that the curcumin can have therapeutic effect against $A D$ through targeting various pathways and proteins.

\section{Protein-Protein Interaction network analysis and key gene identification}

Using the GeneMANIA database, PPI networks were built, visualized and analyzed by Cytoscape software. The PPI data and network is shown in Fig. 4A. Network Analyzer (cytoscope plugin) were utilized to analyse the network topological properties. (Supplementary file 1). Here, the highest degree is 99 and lowest degree is 1 . In order to quantify and visualize the relation between the genes and understand the functions of proteins at the systematic level the PPI network was obtained. The data was then analysed on the basis of the topological parameters like betweenness, degree and closeness and then it was found that APP was present in the centre of the network having the largest degree (degree $=53$ ), closeness (closeness $=0.699$ ) and betweenness (betweenness $=0.06$ ) followed by MAPK1, PPAR $y$, STAT3, CTSD, AGTR1, RARA, MET, STAT1, and KDR. A gene with the higher degree $(k)$ and centralities (betweenness and closeness) value can aid to recognize a biological system having main role in the network. Therefore, we have computed degree $(k)$, centralities betweenness (CB) and closeness (CC) by using NetworkAnalyzer. The details of top ten degree and centrality measurement (CB and CC) of PPI network are given in Fig. 4B, Fig. 4C and Fig 4D respectively. First, we have selected the first10 genes based on ranking of degree, betweenness and closeness. After that find, the common genes in degree, betweenness and closeness of network (Fig. 4E). We identified key genes in regulatory network of AD. Therefore, we have identified five key gene RARA, APP, PRARG, STAT3 and MAPK1 in PPI network (Fig. 4A). These entire key genes play a great role in the progression of AD. The PPI network of 74 genes is shown in Fig. 4A with the key gene highlighted in yellow. The regulatory network of these key gene is given in Fig. $\mathbf{4 F}$

\section{Mapping of targeted $A D$ genes with all brain expressed genes analysis}

SynGO (Koopmans et al., 2019) showed clear enrichment in ontological categories correlated with synaptic signalling and synapse organisation for inhibitory neuron genes found with $A D$ targeted gene. The evaluation of the results indicated that 15 genes like CTSD, APP, and MAPK1 are synaptic and are localised in the synaptic membrane. 10 genes are the presynaptic and are localised in pre-synaptic membrane, endosome, cytosol and exoskeleton. Out of the inputted genes 16 genes were also showing the role in process of synapse. Studies have also been established that CTSD and APP protein promotes synapse formation and neuronal migration. The impairment of CTSD and APP protein can lead to 
dysregulation of synaptic transmission therefore the protein have a part in the patho-physiology of the cerebral degenerative disease [40] [41]. The Fig. 4G below shows the biological function of genes in the synapses.

\section{VarElect Analysis of the Key genes}

For the genotypic-phenotypic analysis of 74 intersection genes were loaded in the VarElect online server. The result is shown in Table 2. Amyloid precursor protein (APP) is a single pass trans-membrane protein belonging to the family of the proteins called amyloid precursor-like proteins (APLP) in Drosophila, APLP1 and APLP2 of mammals. This APP protein is responsible for the lodgement of the neurotoxic $\beta$-amyloid peptide $(A B)$ in the brain which is key factor for the development of AD [42]. In humans, APP gene is present on the chromosome number 21 which translates into three isoforms i.e., APP695, APP770 and APP751. These forms vary on the basis of presence of Kunitz Protease Inhibitor (KPI) domain within their extracellular regions [43]. The APP695 lacks this domain and it has been reported that in AD patients this KPI lacking isoform gets converted to KPI containing isoforms i.e. APP770 and APP751 which is found associated with increased production of $A \beta$ [44]. Another Protein called Cathepsin D (CTSD) is a gene which is involved in the processing of APP protein. The impaired activity of the CTSD protein leads to the accumulation of the $\beta$-amyloid peptide $(A \beta)$ thereby causing the progression of $A D$ [45] [46]. The development of the amyloid peptides causes the inflammatory response in the brain. The peroxisome proliferator-activated receptor gamma (PPAR-gamma) is transcription factor which suppress that inflammatory gene expression by regulating the glucose and lipid metabolism [47] [48].

Table 2: VarElect Analysis of target proteins of Alzheimer with the targets of Curcumin. The score tells the relation between the genes and the target phenotype. 


\begin{tabular}{|c|c|c|c|c|}
\hline Symbol & Description & Category & Score & $\begin{array}{l}\text { Average Disease- } \\
\text { Causing Likelihood }\end{array}$ \\
\hline APP & Amyloid Beta Precursor Protein & Alzheimer & 41.00 & $77.65 \%$ \\
\hline PLAU & Plasminogen Activator, Urokinase & Alzheimer & 28.26 & $32.24 \%$ \\
\hline NOS3 & Nitric Oxide Synthase 3 & Alzheimer & 28.26 & $58.50 \%$ \\
\hline GBA & Glucosylceramidase Beta & Alzheimer & 13.62 & $61.31 \%$ \\
\hline BACE1 & Beta-Secretase 1 & Alzheimer & 6.81 & $78.70 \%$ \\
\hline GSK3B & Glycogen Synthase Kinase 3 Beta & Alzheimer & 6.11 & $83.18 \%$ \\
\hline MAOB & Monoamine Oxidase B & Alzheimer & 5.67 & $84.51 \%$ \\
\hline PPARA & $\begin{array}{l}\text { Peroxisome Proliferator Activated } \\
\text { Receptor Alpha }\end{array}$ & Alzheimer & 5.56 & $36.99 \%$ \\
\hline CTSD & Cathepsin D & Alzheimer & 5.52 & $68.84 \%$ \\
\hline PPAR $y$ & $\begin{array}{l}\text { Peroxisome Proliferator Activated } \\
\text { Receptor Gamma }\end{array}$ & Alzheimer & 5.50 & $58.38 \%$ \\
\hline TTR & Transthyretin & Alzheimer & 5.32 & $43.90 \%$ \\
\hline MME & Membrane Metalloendo peptidase & Alzheimer & 5.26 & $69.79 \%$ \\
\hline AKT1 & AKT Serine/Threonine Kinase 1 & Alzheimer & 5.15 & $90.77 \%$ \\
\hline IL2 & Interleukin 2 & Alzheimer & 5.12 & $75.55 \%$ \\
\hline HTR7 & 5-Hydroxytryptamine Receptor 7 & Alzheimer & 5.12 & $82.77 \%$ \\
\hline ESR1 & Estrogen Receptor 1 & Alzheimer & 5.12 & $68.53 \%$ \\
\hline CASP3 & Caspase 3 & Alzheimer & 5.12 & $64.18 \%$ \\
\hline MAPK8 & Mitogen-Activated Protein Kinase 8 & Alzheimer & 5.09 & $87.00 \%$ \\
\hline MAPK10 & Mitogen-Activated Protein Kinase 10 & Alzheimer & 5.09 & $78.02 \%$ \\
\hline HMOX1 & Heme Oxygenase 1 & Alzheimer & 5.09 & $31.92 \%$ \\
\hline CDK1 & Cyclin Dependent Kinase 1 & Alzheimer & 5.06 & $79.71 \%$ \\
\hline MAPK1 & Mitogen-Activated Protein Kinase 1 & Alzheimer & 5.06 & $73.83 \%$ \\
\hline CDK5R1 & $\begin{array}{l}\text { Cyclin Dependent Kinase } 5 \text { Regulatory } \\
\text { Subunit } 1\end{array}$ & Alzheimer & 5.06 & $88.63 \%$ \\
\hline ALOX5 & Arachidonate 5-Lipoxygenase & Alzheimer & 5.06 & $77.22 \%$ \\
\hline CYP19A1 & $\begin{array}{l}\text { Cytochrome P450 Family } 19 \text { Subfamily } \\
\text { A Member } 1\end{array}$ & Alzheimer & 5.06 & $49.30 \%$ \\
\hline
\end{tabular}




\begin{tabular}{|c|c|c|c|c|}
\hline PTGS2 & Prostaglandin-Endoperoxide Synthase 2 & Alzheimer & 5.06 & $66.54 \%$ \\
\hline REG1A & Regenerating Family Member 1 Alpha & Alzheimer & 5.06 & $82.07 \%$ \\
\hline HMGCR & $\begin{array}{l}\text { 3-Hydroxy-3-Methylglutaryl-CoA } \\
\text { Reductase }\end{array}$ & Alzheimer & 5.06 & $83.06 \%$ \\
\hline REN & Renin & Alzheimer & 5.06 & $75.65 \%$ \\
\hline INSR & Insulin Receptor & Alzheimer & 5.06 & $75.63 \%$ \\
\hline NOS2 & Nitric Oxide Synthase 2 & Alzheimer & 5.06 & $46.59 \%$ \\
\hline EPHA1 & EPH Receptor A1 & Alzheimer & 2.93 & $34.44 \%$ \\
\hline AGTR1 & Angiotensin II Receptor Type 1 & Alzheimer & 2.60 & $63.82 \%$ \\
\hline ADH1C & $\begin{array}{l}\text { Alcohol Dehydrogenase 1C (Class I), } \\
\text { Gamma Polypeptide }\end{array}$ & Alzheimer & 2.05 & $0.00 \%$ \\
\hline S100A9 & S100 Calcium Binding Protein A9 & Alzheimer & 1.69 & $67.27 \%$ \\
\hline HTR1A & 5-Hydroxytryptamine Receptor $1 \mathrm{~A}$ & Alzheimer & 0.72 & $56.88 \%$ \\
\hline MAOA & Monoamine Oxidase A & Alzheimer & 0.62 & $84.12 \%$ \\
\hline DRD4 & Dopamine Receptor D4 & Alzheimer & 0.62 & $0.00 \%$ \\
\hline HSP90AA1 & $\begin{array}{l}\text { Heat Shock Protein } 90 \text { Alpha Family } \\
\text { Class A Member } 1\end{array}$ & Alzheimer & 0.51 & $79.59 \%$ \\
\hline ESR2 & Estrogen Receptor 2 & Alzheimer & 0.51 & $54.14 \%$ \\
\hline MMP9 & Matrix Metallopeptidase 9 & Alzheimer & 0.36 & $20.28 \%$ \\
\hline PARP1 & Poly (ADP-Ribose) Polymerase 1 & Alzheimer & 0.36 & $45.76 \%$ \\
\hline PDE5A & Phosphodiesterase 5A & Alzheimer & 0.36 & $29.70 \%$ \\
\hline ALB & Albumin & Alzheimer & 0.36 & $82.33 \%$ \\
\hline SOD2 & Superoxide Dismutase 2 & Alzheimer & 0.36 & $35.48 \%$ \\
\hline NFE2L2 & Nuclear Factor, Erythroid 2 Like 2 & Alzheimer & 0.36 & $54.66 \%$ \\
\hline
\end{tabular}

\section{Spatiotemporal expression patterns of five key genes in various regions of human brain}

Additionally, to test if the key genes PPAR $y$, MAPK1, STAT3, RARA and APP causes the risk of AD, we searched the expression profiling of PPAR $\gamma$, MAPK1, STAT3, RARA and APP in different regions human brain tissues utilizing the BRAINEAC data (Fig.5). We identified that PPAR $y$, MAPK1, STAT3, RARA and APP are expressed differently in various brain regions, with the highest transcript level was observed for MAPK1 in the putamen, followed by hippocampus, temporal cortex, frontal cortex, occipital cortex and thalamus (Fig. 5B). The least expression was observed in cerebral cortex for PPAR $y$, RARA and APP. Both RARA and PPAR $y$ showed highest expression in the thalamus. 
In order to evaluate comparative temporospatial expression of PPAR $y$, MAPK1, STAT3, RARA and APP in human central nervous system (CNS), we searched the Human Brain Transcriptome (HBT) dataset (https://hbatlas.org/), a database based on Affymetrix GeneChip arrays [49]. As it is seen in Fig 6, PPARY, MAPK1, STAT3, RARA and APP mRNA expression is consistent from conception to adulthood in all brain regions shown here which includes: mediodorsal nucleus of the thalamus (MD), cerebellar cortex (CBC), amygdala region (AMY), striatum (STR), hippocampus (HIP) and neocortex (NCX)The highest expression was seen for APP followed by MAPK1 and STAT3. The temporal expression analysis showed that in the case of RARA the expression level was high during the fetal development, but the expression level gradually decreased as the human brain developed. The trajectory plot of PPARY further tells that the expression of PPAR $y$ mRNA expression is quite uniform from conception to adulthood in all depicted brain regions, including NCX, AMY, STR, MD, and CBC. All regions indicate comparatively lower level of expression in early embryonal life which slowly increases during fetal development till birth and increase again postnatal life and early childhood up to adolescence (Fig. 6), which remains quite stable thereafter. It must be noted however, that PPAR $y$ expression in hippocampal region (blue line) behaves differently indicating a more significant rise in early postnatal life and persisting at higher levels (in comparison to other tissues) up to adulthood.

\section{Molecular Docking}

Molecular docking studies helped us in better understanding the binding interactions of the key proteins with the ligand molecule. The molecular docking of key proteins RARA, APP, PPAR $y$, STAT3 and MAPK1 with the curcumin was carried out using Glide. The glide score and glide binding energy for all the key genes are shown in the Table 3. The results showed that the stronger interaction is present between PPAR $y$ molecule and curcumin having Glide energy $49.03 \mathrm{kcal} / \mathrm{mol}$ and glide score as -7.269. The 2-D structure of the docking complex reveals that Curcumin interacts with the amino acids GLU 295 in the active site (Fig. 7). The interacting amino acids of the various docking complex is given in (Table 3 ).

Table 3: Molecular Docking analysis results of Curcumin and shortlisted targets of protein showing Glide Score, energy of the docking and interacting amino acids 


\begin{tabular}{|c|c|c|c|}
\hline $\begin{array}{l}\text { Target } \\
\text { Proteins }\end{array}$ & $\begin{array}{l}\text { Glide } \\
\text { energy } \\
\text { (Kcal/mol) }\end{array}$ & $\begin{array}{l}\text { Glide } \\
\text { Score }\end{array}$ & Interacting Amino acids \\
\hline PPARY & -49.03 & -7.269 & $\begin{array}{l}\text { PHE 282, CYS 285, GLN 286, ARG 288, SER289, GLU 291, ALA } \\
\text { 292, GLU 295, VAL 339, LEU 340, ILE 341, SER342, HIE 449, PHE } \\
\text { 226, HIE 323, ILE 325, ILE 326, TYR 327, MET 329, LEU 330, } \\
\text { LEU333 }\end{array}$ \\
\hline MAPK1 & -43.637 & -5.635 & $\begin{array}{l}\text { VAL 37, ALA 33, ASP109, GLY32, GLU 31, LLE 29, MET 106, } \\
\text { LEU105, ASP104, GLN 103, ALA 50, LYS 52, LEU154, ASN 152, } \\
\text { LYS 149, ASP 147, ARG 65, THR188, LEU168, ASP 165, CYS 164, } \\
\text { GLU } 69\end{array}$ \\
\hline STAT3 & -43.489 & -5.329 & $\begin{array}{l}\text { ILE 258, ASN257, PRO 256, GLY 253, CYS 251, ALA 250, GLN 247, } \\
\text { SER 514, TRP 474, PRO 336, ASP 334, PRO 333, ARG 325, GLN } \\
\text { 326, CYS } 328\end{array}$ \\
\hline RARA & -34.122 & -5.312 & $\begin{array}{l}\text { TRP 225, PHE 228, SER 229, SER 232, PRO 407, PRO 408, LEU } \\
\text { 409, ILE 410, GLN 411, VAL 395, ALA 392, ALA 389, SER 388, ARG } \\
\text { 385, LYS 262, ALA 263, CYS 265, LEU 266, ASP 267, LEU 269, ARG } \\
339\end{array}$ \\
\hline APP & -36.255 & -3.980 & $\begin{array}{l}\text { LYS 314, LEU 311, PHE } 310, \text { HIS 307, VAL } 373 \text {, HIS } 376, \text { ALA } 377, \\
\text { VAL 380, ILE 381, VAL 434, HIS 433, HIS 430, ARG 429, HIS } 426\end{array}$ \\
\hline
\end{tabular}

\section{Discussion}

Due to poor understanding of the mechanism and sudden emergence of the Alzheimer have made it difficult to identify the cause and prevent the progression of disease. The main mechanism of action of the drugs available in the market is to ease improve the cognitive function and health of the patient [50]. Curcumin a natural plant derived molecule with anti-inflammatory and antioxidant function helps in the reduction of amyloid aggregation which is considered major factors of the cognitive impairment in the $A D$ patients [51]. Despite of many clinical and experimental studies the precise molecular mechanism of curcumin against $A D$ is not clearly understood. Using an integrative approach of network pharmacology with target mining, drug likeness, enrichment analysis, protein-protein interaction and docking the efforts were made to analyse the therapeutic mechanism of curcumin against AD.

The curcumin was checked for the drug-likeness using the Lipinski's R05 parameters, the results showed that curcumin with 0.55 bioavailability score has good pharmacokinetic properties and is a potential therapeutic oral drug. Then the targets of curcumin and genes related to $A D$ were obtained using multiple databases. From the targets set of curcumin 74 targets were selected for AD and VarElect analysis was performed on these genes. The analysis was performed to find the correlation between the phenotypes of $A D$ and the possible target genes. Amyloid precursor protein (APP) and Beta-secretase 1 (BACE1) was the highest scored among the possible targets with approximately $78 \%$ of the probability of causing disease. The human APP is related to a family of Amyloid like proteins called APLP1 and APLP2 (in mammals) 
and APPL (in drosophila). The studies done on genetic analyses of few organisms with one APP members have suggested the role of the protein in neuronal development. The APPL knockout drosophila have shown defects at neuromuscular junction and affecting the Wnt-planar cell polarity pathway (PCP) pathway which plays role in neuronal development [52] [53]. APP protein undergoes sequential protein cleavage by the enzymes a-secretase and b-secretase resulting in non-amyloidogenic and amyloidogenic fragments. But the processing at the b-site by the b-secretase enzyme(BACE1) which results in the amyloidogenic fragments which are said to be associated with $A D$ pathology as they result in the formation of $\beta$-amyloid peptides (AB) which are major hallmarks in the AD [54] [43]. These two genes are main targets for the treatment of $A D$. Another highly scored protein called Cathepsin $D$ (CTSD) protein shows approximately $70 \%$ probability of causing AD. It is lysosomal protease which is responsible for the degradation of the amyloid plaques. It was reported that the levels of CTSD protein down regulated in the $A D$ patients and is said to play a role in the deposition of the amyloid plaques in the cortex of the brain. Therefore the levels of Cathepsin D may be used as a diagnostic biomarker for AD [55]. Another Protein signalling called retinoic acid receptor (RAR)a signalling is reported to increase the expression of metalloprotease 10 and disintegrins that process the amyloid precursor protein and prevent its accumulation thereby preventing neuronal cell death associated with $A \beta[56]$.

Enrichment analysis of the targets was also conducted using Enrichr and Metascape then the networks of the protein-protein interaction were carried out by Cytoscape 3.6. The target genes shows that they played an important role in $\mathrm{GO}$ biological processes that are mostly involved in regulation of inflammatory responses, regulation of protein phosphorylation and peptidyl-tyrosine modification. It is well established that due to disturbance in the balance of pro-inflammatory and anti-inflammatory signalling in the $A D$ patients the chronic inflammation occurs which further aggravates the pathogenesis [57]. Another hallmark for the AD is the neurofibrillary tangles (NFTs) which are formed due to hyperphosphorylation of the tau proteins at the multiple sites which results in the disintegration of the microtubule structure and disturbance of cellular processes [58] [59]. Thus, regulation of these inflammatory signalling and protein phosphorylation is an important way to manage AD. Lipooxygenase (LOX), Cyclooxygenase (COX-2), and Inducible nitric oxide Synthase (iNOS) are important enzymes that regulate inflammatory processes. It is specified that curcumin possess anti-inflammatory action by hindering the Toll-like receptor 4-dependent, COX-2, LOX, iNOS and nuclear factor-kappaB signalling pathways and the activation of a peroxisome proliferator-activated receptor-gamma pathway (PPARY) [60] [61]. Inflammation is also closely linked with the tumour formation therefore the curcumin shows chemo preventive role in the cancer formation [61]. PPARy agonists have been reported to inhibit neuroinflammation and reducing amyloid and tau pathologies in few animal models and in the patients with mild $A D$ but the molecular mechanism is not thoroughly investigated [48]. It was studied that the extracellular signal-regulated protein kinase (ERK MAPK) signalling is important for various forms of hippocampus-dependent memory and learning that are hindered in AD. Based on these studies it was further showed that inhibition of PPARY prevents the recruitment of PPARY to ERK which showed that formation of this protein complex is requisite for memory formation[62]. 
Synaptic plasticity changes are mainly reported as an important basic principles of cognitive processes, such as memory formation, sensory processing, attention, associative learning, and decision making and retrieval, perception. Loss of synaptic plasticity or its dysregulation is considered major cause of Dementia or $\mathrm{AD}[35]$. The resources lack a comprehensive ontology of synaptic processes and locations so in order to overcome these shortcomings another tool called SynGO was used for ontology of synaptic genes. $A D$ is distinguished by hippocampal-dependent dysfunction, cathepsins are also important for hippocampal-dependent learning and memory. It is reported that, cathepsins on being secreted in the extracellular space produces extracellular effects on synaptic plasticity and neuroprotection thereby playing role in $\mathrm{AD}[40]$. The results of Enrichment analysis were also found compatible with the correlation between targets and phenotypes in VarElect Analysis.

Protein-protein interaction was constructed to understand the significance of curcumin targets. The Cytoscape tool was used to identify top five key genes namely, PPARY, MAPK1, STAT3, KDR and APP. Further the expression profiling of these key genes in different human brain tissues was done utilizing the BRAINEAC data and comparative temporospatial expression human central nervous system (CNS), was done by exploring the Human Brain Transcriptome (HBT) dataset. At last, molecular docking was used to investigate the mechanism of interactions between curcumin and five key targets. The results indicated that PPAR $y$, MAPK1, STAT3, KDR and APP showed affinity with Curcumin. The results showed that the Curcumin has potential to bind with large active sites of PPARy with good binding scores compared to all other proteins. Therefore, the precise mechanism of Curcumin on AD based on molecular docking need further validation using biological experiments.

\section{Conclusion}

This study provided a novel method to disclose the therapeutic mechanisms of Curcumin against AD. The results indicated that Curcumin may have an anti-AD effect through multiple targets, pathways, and biological processes. However, more future studies are required to confirm the precise mechanism and the clinical potential and mechanisms of curcumin against AD.

\section{Declarations}

Not Applicable.

\section{Acknowledgement}

A. I. acknowledge University Grant Commission (UGC) for Maulana Azad National Fellowship (candidate ID: MANF-2018-19-BIH-93364).

\section{Author contributions}

M.Z.M. conceived the model. D.A. A.I. and MZ.M. prepared figures of the numerical results. D.A. A.I. M.M.U.H. and M.Z.M. analysed and interpreted the results, D.A. A.I., M.M.U.H., A.I. and MZ.M wrote the 
manuscript. A.I and M.Z.M. supervised the study and approved the final draft.

\section{Competing interests}

The authors declare no competing interests.

\section{Availability of data and materials}

The data used in the current study available from the corresponding author on reasonable request.

\section{Ethics approval and consent to participate.}

Not Applicable.

\section{Consent for publication}

Not Applicable.

\section{References}

1. “2020 Alzheimer's disease facts and figures - 2020 - Alzheimer's \&amp; Dementia - Wiley Online Library," Alzehimer's Association. https://alzjournals.onlinelibrary.wiley.com/doi/full/10.1002/alz.12068 (accessed Oct. 22, 2021).

2. C. Calabrese, W. L. Gregory, M. Leo, D. Kraemer, K. Bone, and B. Oken, "Effects of a Standardized Bacopa monnieri Extract on Cognitive Performance, Anxiety, and Depression in the Elderly: A Randomized, Double-Blind, Placebo-Controlled Trial," J. Altern. Complement. Med., vol. 14, no. 6, pp. 707-713, Jul. 2008, doi: 10.1089/acm.2008.0018.

3. C. Franchi et al., "Cholinesterase inhibitor use in Alzheimer's disease: the EPIFARM-Elderly Project," Pharmacoepidemiol. Drug Saf., vol. 20, no. 5, pp. 497-505, May 2011, doi: 10.1002/pds. 2124.

4. K.-X. Dou et al., "Comparative safety and effectiveness of cholinesterase inhibitors and memantine for Alzheimer's disease: a network meta-analysis of 41 randomized controlled trials," Alzheimers Res. Ther., vol. 10, no. 1, p. 126, Dec. 2018, doi: 10.1186/s13195-018-0457-9.

5. R. V. Rao, O. Descamps, V. John, and D. E. Bredesen, “Ayurvedic medicinal plants for Alzheimer's disease: a review," Alzheimers Res. Ther., vol. 4, no. 3, p. 22, Jun. 2012, doi: 10.1186/alzrt125.

6. A. A. Farooqui, T. Farooqui, A. Madan, J. H.-J. Ong, and W.-Y. Ong, "Ayurvedic Medicine for the Treatment of Dementia: Mechanistic Aspects," Evid. Based Complement. Alternat. Med., vol. 2018, p. e2481076, May 2018, doi: 10.1155/2018/2481076.

7. J. Mehla, P. Gupta, M. Pahuja, D. Diwan, and D. Diksha, "Indian Medicinal Herbs and Formulations for Alzheimer's Disease, from Traditional Knowledge to Scientific Assessment," Brain Sci., vol. 10, no. 12, p. E964, Dec. 2020, doi: 10.3390/brainsci10120964.

8. G. P. Lim, T. Chu, F. Yang, W. Beech, S. A. Frautschy, and G. M. Cole, "The Curry Spice Curcumin Reduces Oxidative Damage and Amyloid Pathology in an Alzheimer Transgenic Mouse," J. Neurosci., 
vol. 21, no. 21, pp. 8370-8377, Nov. 2001, doi: 10.1523/JNEUROSCl.21-21-08370.2001.

9. F. Yang et al., "Curcumin inhibits formation of amyloid beta oligomers and fibrils, binds plaques, and reduces amyloid in vivo," J. Biol. Chem., vol. 280, no. 7, pp. 5892-5901, Feb. 2005, doi: 10.1074/jbc.M404751200.

10. S. I. Berger and R. lyengar, "Network analyses in systems pharmacology,” Bioinformatics, vol. 25, no. 19, pp. 2466-2472, Oct. 2009, doi: 10.1093/bioinformatics/btp465.

11. A. Daina, O. Michielin, and V. Zoete, "SwissADME: a free web tool to evaluate pharmacokinetics, druglikeness and medicinal chemistry friendliness of small molecules," Sci. Rep., vol. 7, no. 1, p. 42717, Mar. 2017, doi: 10.1038/srep42717.

12. A. Daina and V. Zoete, "A BOILED-Egg To Predict Gastrointestinal Absorption and Brain Penetration of Small Molecules," ChemMedChem, vol. 11, no. 11, pp. 1117-1121, Jun. 2016, doi: 10.1002/cmdc.201600182.

13. M. Abdul-Hammed et al., "Virtual screening, ADMET profiling, PASS prediction, and bioactivity studies of potential inhibitory roles of alkaloids, phytosterols, and flavonoids against COVID-19 main protease (Mpro)," Nat. Prod. Res., pp. 1-7, Jun. 2021, doi: 10.1080/14786419.2021.1935933.

14. T. Liu, Y. Lin, X. Wen, R. N. Jorissen, and M. K. Gilson, “BindingDB: a web-accessible database of experimentally determined protein-ligand binding affinities," Nucleic Acids Res., vol. 35, no. Database issue, pp. D198-201, Jan. 2007, doi: 10.1093/nar/gkl999.

15. X. Wang et al., "PharmMapper 2017 update: a web server for potential drug target identification with a comprehensive target pharmacophore database," Nucleic Acids Res., vol. 45, no. W1, pp. W356W360, Jul. 2017, doi: 10.1093/nar/gkx374.

16. M. Kuhn, D. Szklarczyk, A. Franceschini, C. von Mering, L. J. Jensen, and P. Bork, "STITCH 3: zooming in on protein-chemical interactions," Nucleic Acids Res., vol. 40, no. Database issue, pp. D876-D880, Jan. 2012, doi: 10.1093/nar/gkr1011.

17. L. Yang et al., "Systematic Elucidation of the Mechanism of Quercetin against Gastric Cancer via Network Pharmacology Approach," BioMed Res. Int., vol. 2020, p. e3860213, Sep. 2020, doi: $10.1155 / 2020 / 3860213$.

18. G. Stelzer et al., "VarElect: the phenotype-based variation prioritizer of the GeneCards Suite," $B M C$ Genomics, vol. 17, no. 2, p. 444, Jun. 2016, doi: 10.1186/s12864-016-2722-2.

19. R. Barshir et al., "GeneCaRNA: A Comprehensive Gene-centric Database of Human Non-coding RNAs in the GeneCards Suite," J. Mol. Biol., vol. 433, no. 11, p. 166913, May 2021, doi: 10.1016/j.jmb.2021.166913.

20. Y. Chen et al., "Systematic Elucidation of the Mechanism of Genistein against Pulmonary Hypertension via Network Pharmacology Approach," Int. J. Mol. Sci., vol. 20, no. 22, Art. no. 22, Jan. 2019, doi: 10.3390/ijms20225569.

21. O. Kononenko, O. Baysal, R. Holmes, and M. W. Godfrey, "Mining modern repositories with elasticsearch," in Proceedings of the 11th Working Conference on Mining Software Repositories, New York, NY, USA, May 2014, pp. 328-331. doi: 10.1145/2597073.2597091. 
22. Z. Ding and D. Kihara, "Computational identification of protein-protein interactions in model plant proteomes," Sci. Rep., vol. 9, no. 1, p. 8740, Jun. 2019, doi: 10.1038/s41598-019-45072-8.

23. H. Lu et al., "Recent advances in the development of protein-protein interactions modulators: mechanisms and clinical trials," Signal Transduct. Target. Ther., vol. 5, no. 1, p. 213, Sep. 2020, doi: 10.1038/s41392-020-00315-3.

24. P. Shannon et al., "Cytoscape: A Software Environment for Integrated Models of Biomolecular Interaction Networks," Genome Res., vol. 13, no. 11, pp. 2498-2504, Nov. 2003, doi: 10.1101/gr.1239303.

25. S. Ali, M. Z. Malik, S. S. Singh, K. Chirom, R. Ishrat, and R. K. B. Singh, “Exploring novel key regulators in breast cancer network," PLOS ONE, vol. 13, no. 6, p. e0198525, Jun. 2018, doi: 10.1371/journal.pone.0198525.

26. Y. Assenov, F. Ramírez, S.-E. Schelhorn, T. Lengauer, and M. Albrecht, "Computing topological parameters of biological networks," Bioinforma. Oxf. Engl., vol. 24, no. 2, pp. 282-284, Jan. 2008, doi: 10.1093/bioinformatics/btm554.

27. M. E. J. Newman and M. Girvan, "Finding and evaluating community structure in networks," Phys. Rev. E, vol. 69, no. 2, p. 026113, Feb. 2004, doi: 10.1103/PhysRevE.69.026113.

28. "A faster algorithm for betweenness centrality: The Journal of Mathematical Sociology: Vol 25, No 2." https://www.tandfonline.com/doi/abs/10.1080/0022250X.2001.9990249 (accessed Jan. 12, 2022).

29. A. Farooqui et al., "Assessment of the key regulatory genes and their Interologs for Turner Syndrome employing network approach," Sci. Rep., vol. 8, no. 1, p. 10091, Jul. 2018, doi: 10.1038/s41598-01828375-0.

30. A. Subramanian et al., "Gene set enrichment analysis: a knowledge-based approach for interpreting genome-wide expression profiles," Proc. Natl. Acad. Sci. U. S. A., vol. 102, no. 43, pp. 15545-15550, Oct. 2005, doi: 10.1073/pnas.0506580102.

31. H. Tipney and L. Hunter, "An introduction to effective use of enrichment analysis software," Hum. Genomics, vol. 4, no. 3, p. 202, Feb. 2010, doi: 10.1186/1479-7364-4-3-202.

32. M. V. Kuleshov et al., "Enrichr: a comprehensive gene set enrichment analysis web server 2016 update," Nucleic Acids Res., vol. 44, no. W1, pp. W90-W97, Jul. 2016, doi: 10.1093/nar/gkw377.

33. Y. Zhou et al., "Metascape provides a biologist-oriented resource for the analysis of systems-level datasets," Nat. Commun., vol. 10, no. 1, p. 1523, Apr. 2019, doi: 10.1038/s41467-019-09234-6.

34. H. R. Monday and P. E. Castillo, "Closing the gap: long-term presynaptic plasticity in brain function and disease," Curr. Opin. Neurobiol., vol. 45, pp. 106-112, Aug. 2017, doi: 10.1016/j.conb.2017.05.011.

35. F. Koopmans et al., "SynGO: An Evidence-Based, Expert-Curated Knowledge Base for the Synapse," Neuron, vol. 103, no. 2, pp. 217-234.e4, Jul. 2019, doi: 10.1016/j.neuron.2019.05.002.

36. L. M. F. Sng, P. C. Thomson, and D. Trabzuni, "Genome-wide human brain eQTLs: In-depth analysis and insights using the UKBEC dataset," Sci. Rep., vol. 9, no. 1, p. 19201, Dec. 2019, doi: 
10.1038/s41598-019-55590-0.

37. R. A. Friesner et al., "Glide: a new approach for rapid, accurate docking and scoring. 1. Method and assessment of docking accuracy," J. Med. Chem., vol. 47, no. 7, pp. 1739-1749, Mar. 2004, doi: 10.1021/jm0306430.

38. J. Bojarska et al., "A Supramolecular Approach to Structure-Based Design with A Focus on Synthons Hierarchy in Ornithine-Derived Ligands: Review, Synthesis, Experimental and in Silico Studies," Molecules, vol. 25, no. 5, Art. no. 5, Jan. 2020, doi: 10.3390/molecules25051135.

39. L. Kirouac, A. J. Rajic, D. H. Cribbs, and J. Padmanabhan, "Activation of Ras-ERK Signaling and GSK3 by Amyloid Precursor Protein and Amyloid Beta Facilitates Neurodegeneration in Alzheimer's Disease," eNeuro, vol. 4, no. 2, p. ENEURO.0149-16.2017, Mar. 2017, doi: 10.1523/ENEURO.014916.2017.

40. C. Niemeyer, N. Matosin, D. Kaul, A. Philipsen, and N. C. Gassen, "The Role of Cathepsins in Memory Functions and the Pathophysiology of Psychiatric Disorders," Front. Psychiatry, vol. 11, p. 718, 2020, doi: $10.3389 /$ fpsyt.2020.00718.

41. D. Hefter, S. Ludewig, A. Draguhn, and M. Korte, "Amyloid, APP, and Electrical Activity of the Brain," Neurosci. Rev. J. Bringing Neurobiol. Neurol. Psychiatry, vol. 26, no. 3, pp. 231-251, Jun. 2020, doi: $10.1177 / 1073858419882619$.

42. R. J. O'Brien and P. C. Wong, "Amyloid Precursor Protein Processing and Alzheimer's Disease," Annu. Rev. Neurosci., vol. 34, pp. 185-204, 2011, doi: 10.1146/annurev-neuro-061010-113613.

43. J. Kang and B. Müller-Hill, "Differential splicing of Alzheimer's disease amyloid A4 precursor RNA in rat tissues: PreA4(695) mRNA is predominantly produced in rat and human brain," Biochem. Biophys. Res. Commun., vol. 166, no. 3, pp. 1192-1200, Feb. 1990, doi: 10.1016/0006-291x(90)90992-v.

44. K. Bordji, J. Becerril-Ortega, O. Nicole, and A. Buisson, "Activation of extrasynaptic, but not synaptic, NMDA receptors modifies amyloid precursor protein expression pattern and increases amyloid- $\beta$ production," J. Neurosci. Off. J. Soc. Neurosci., vol. 30, no. 47, pp. 15927-15942, Nov. 2010, doi: 10.1523/JNEUROSCI.3021-10.2010.

45. F. Di Domenico, A. Tramutola, and M. Perluigi, "Cathepsin D as a therapeutic target in Alzheimer's disease," Expert Opin. Ther. Targets, vol. 20, no. 12, pp. 1393-1395, Dec. 2016, doi: 10.1080/14728222.2016.1252334.

46. M. Schuur et al., "Cathepsin D gene and the risk of Alzheimer's disease: A population-based study and meta-analysis," Neurobiol. Aging, vol. 32, no. 9, pp. 1607-1614, Sep. 2011, doi: 10.1016/j.neurobiolaging.2009.10.011.

47. G. Landreth, Q. Jiang, S. Mandrekar, and M. Heneka, "PPARgamma agonists as therapeutics for the treatment of Alzheimer's disease," Neurother. J. Am. Soc. Exp. Neurother., vol. 5, no. 3, pp. 481-489, Jul. 2008, doi: 10.1016/j.nurt.2008.05.003.

48. M. Govindarajulu, P. D. Pinky, J. Bloemer, N. Ghanei, V. Suppiramaniam, and R. Amin, "Signaling Mechanisms of Selective PPARy Modulators in Alzheimer's Disease," PPAR Res., vol. 2018, p. e2010675, Oct. 2018, doi: 10.1155/2018/2010675. 
49. J. M. Keil, A. Qalieh, and K. Y. Kwan, "Brain Transcriptome Databases: A User's Guide,” J. Neurosci., vol. 38, no. 10, pp. 2399-2412, Mar. 2018, doi: 10.1523/JNEUROSCI.1930-17.2018.

50. P. Dallemagne and C. Rochais, "Facing the complexity of Alzheimer's disease," Future Med. Chem., vol. 12, no. 3, pp. 175-177, Feb. 2020, doi: 10.4155/fmc-2019-0310.

51. S. D. Voulgaropoulou, T. a. M. J. van Amelsvoort, J. Prickaerts, and C. Vingerhoets, "The effect of curcumin on cognition in Alzheimer's disease and healthy aging: A systematic review of pre-clinical and clinical studies," Brain Res., vol. 1725, p. 146476, Dec. 2019, doi:

10.1016/j.brainres.2019.146476.

52. L. Torroja, M. Packard, M. Gorczyca, K. White, and V. Budnik, "The Drosophila beta-amyloid precursor protein homolog promotes synapse differentiation at the neuromuscular junction,” J. Neurosci. Off. J. Soc. Neurosci., vol. 19, no. 18, pp. 7793-7803, Sep. 1999.

53. A. Soldano et al., "The Drosophila homologue of the amyloid precursor protein is a conserved modulator of Wnt PCP signaling," PLoS Biol., vol. 11, no. 5, p. e1001562, 2013, doi: 10.1371/journal.pbio.1001562.

54. H. Zheng and E. H. Koo, "Biology and pathophysiology of the amyloid precursor protein," Mol. Neurodegener., vol. 6, no. 1, p. 27, Apr. 2011, doi: 10.1186/1750-1326-6-27.

55. J.-W. Kim et al., "Identification of Cathepsin D as a Plasma Biomarker for Alzheimer's Disease,” Cells, vol. 10, no. 1, p. 138, Jan. 2021, doi: 10.3390/cells10010138.

56. C. I. Jarvis et al., "Retinoic acid receptor-a signalling antagonizes both intracellular and extracellular amyloid- $\beta$ production and prevents neuronal cell death caused by amyloid- $\beta$," Eur. J. Neurosci., vol. 32, no. 8, pp. 1246-1255, Oct. 2010, doi: 10.1111/j.1460-9568.2010.07426.x.

57. S. T. Ferreira, J. R. Clarke, T. R. Bomfim, and F. G. De Felice, “Inflammation, defective insulin signaling, and neuronal dysfunction in Alzheimer's disease," Alzheimers Dement. J. Alzheimers Assoc., vol. 10, no. 1 Suppl, Art. no. 1 Suppl, Feb. 2014, doi: 10.1016/j.jalz.2013.12.010.

58. T. Guo, W. Noble, and D. P. Hanger, "Roles of tau protein in health and disease," Acta Neuropathol. (Berl.), vol. 133, no. 5, pp. 665-704, May 2017, doi: 10.1007/s00401-017-1707-9.

59. J. W. Kinney, S. M. Bemiller, A. S. Murtishaw, A. M. Leisgang, A. M. Salazar, and B. T. Lamb, "Inflammation as a central mechanism in Alzheimer's disease," Alzheimers Dement. Transl. Res. Clin. Interv., vol. 4, pp. 575-590, Sep. 2018, doi: 10.1016/j.trci.2018.06.014.

60. K. Shimizu et al., "Anti-inflammatory Action of Curcumin and Its Use in the Treatment of Lifestylerelated Diseases," Eur. Cardiol., vol. 14, no. 2, pp. 117-122, Jul. 2019, doi: 10.15420/ecr.2019.17.2.

61. V. P. Menon and A. R. Sudheer, "Antioxidant and anti-inflammatory properties of curcumin," Adv. Exp. Med. Biol., vol. 595, pp. 105-125, 2007, doi: 10.1007/978-0-387-46401-5_3.

62. J. B. Jahrling, C. M. Hernandez, L. Denner, and K. T. Dineley, "PPARY Recruitment to Active ERK during Memory Consolidation Is Required for Alzheimer's Disease-Related Cognitive Enhancement," J. Neurosci., vol. 34, no. 11, pp. 4054-4063, Mar. 2014, doi: 10.1523/JNEUROSCI.4024-13.2014.

\section{Figures}




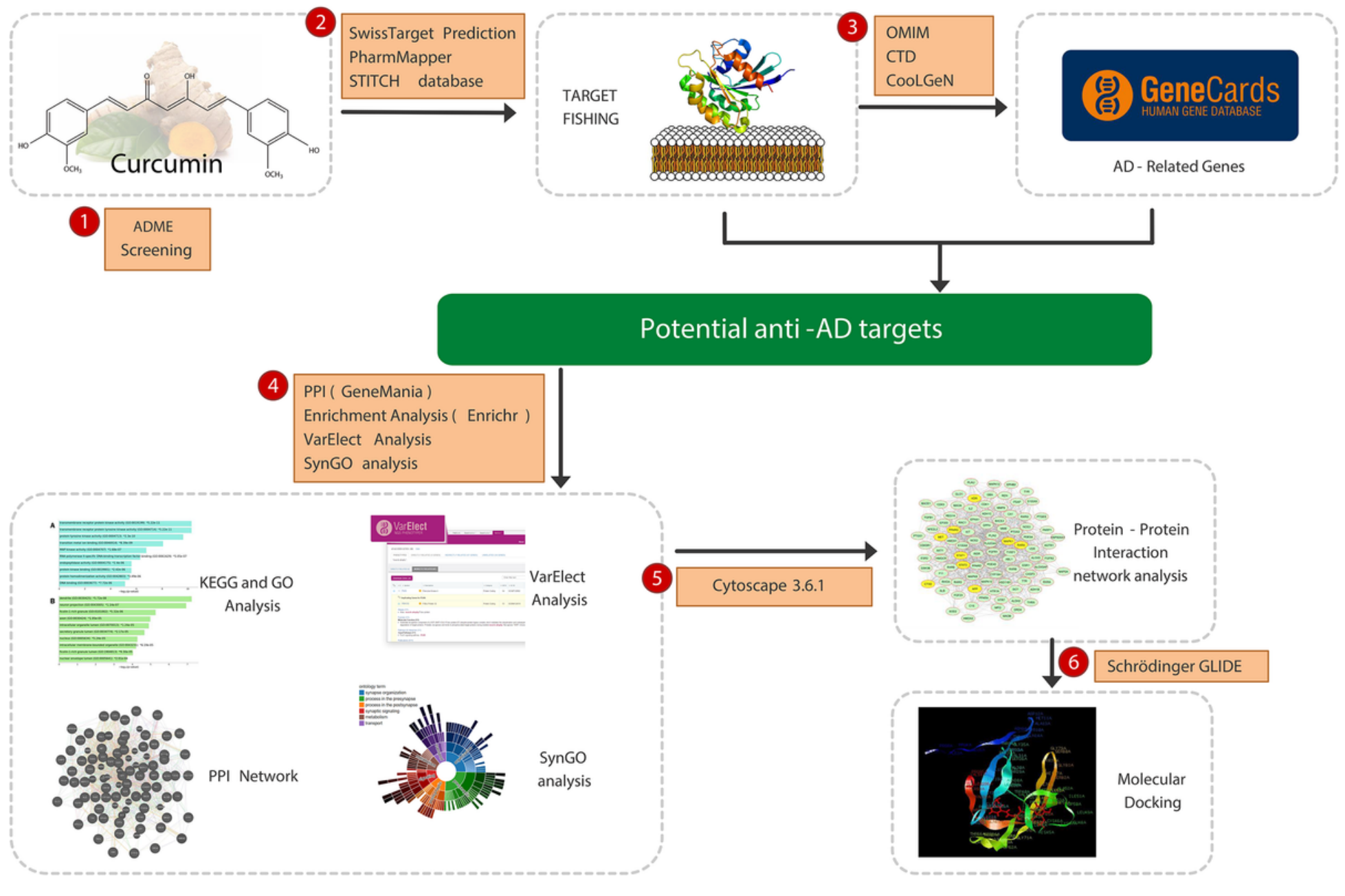

Figure 1

Workflow of the work 

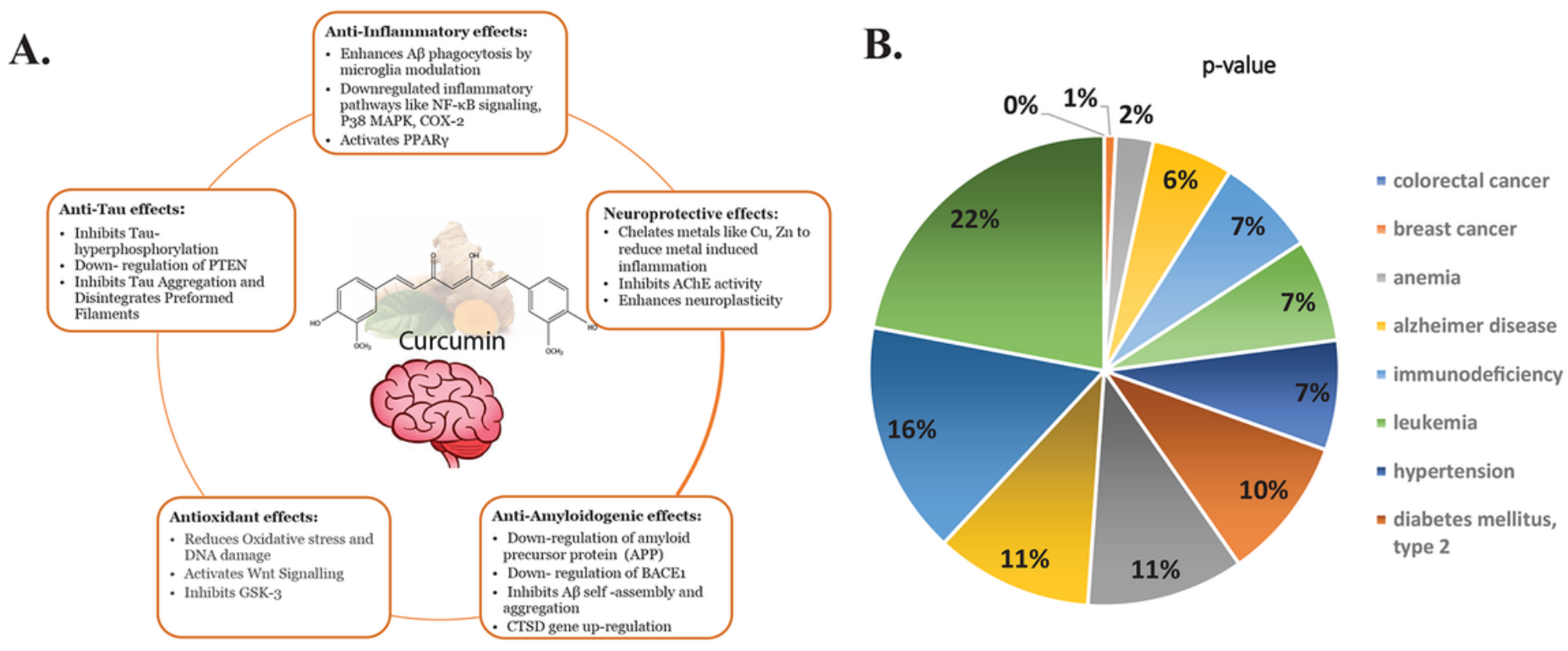

C.

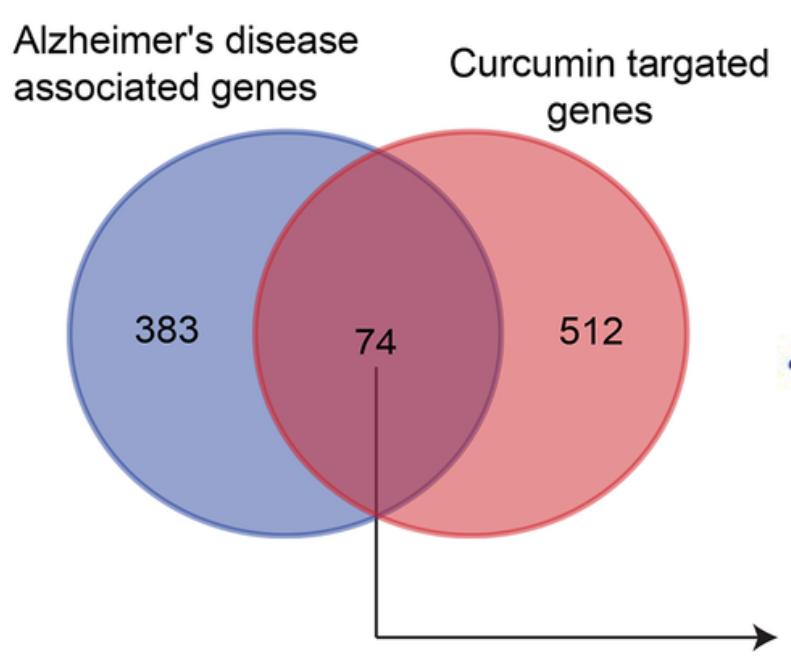

D.

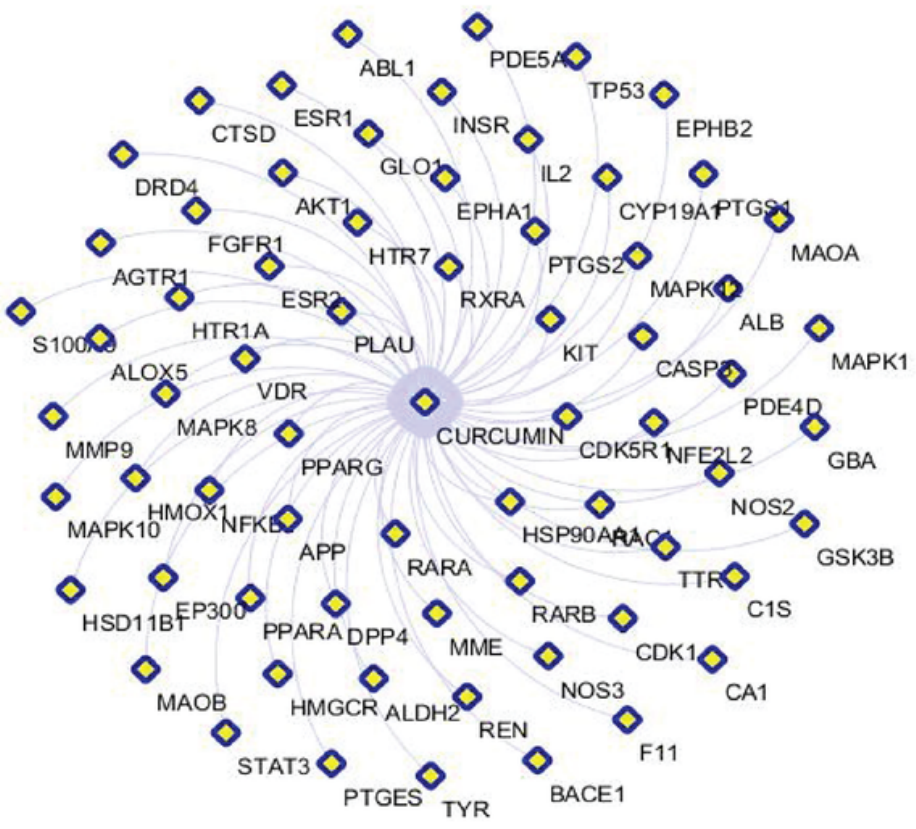

Figure 2

(A) Molecular action of Curcumin in the Alzheimer's disease (AD). (B) Curcumin targeted genes and their role pathway of different diseases. (C) 74 genes selected from the intersection of group of Curcumin targets and AD related targets. (D) Network between Curcumin and target genes. 
A transmembrane receptor protein kinase activity (GO:0019199) *5.22e-11

transmembrane receptor protein tyrosine kinase activity $(60: 0004714) * 5.22 \mathrm{e}-11$ protein tyrosine kinase activity (GO:0004713) $\cdot 2.3 \mathrm{e}-10$ transition metal ion binding $(60: 0046914) * 8.39 \mathrm{e}-09$ MAP kinase activity $(60: 0004707) * 1.68 \mathrm{e}-07$ RNA polymerase II-specific DNA-binding transcription factor binding (GO:0061629) *5.05e-07 endopeptidase activity $(60: 0004175) \cdot 2.4 \mathrm{e}-06$ protein kinase binding (GO:0019901) *2.42e-06 protein homodimerization activity (GO:0042803) *3.49e-06 DNA binding (GO:0003677) $\cdot 7.72 \mathrm{e}-06$

\section{-logio(p-value)}

B

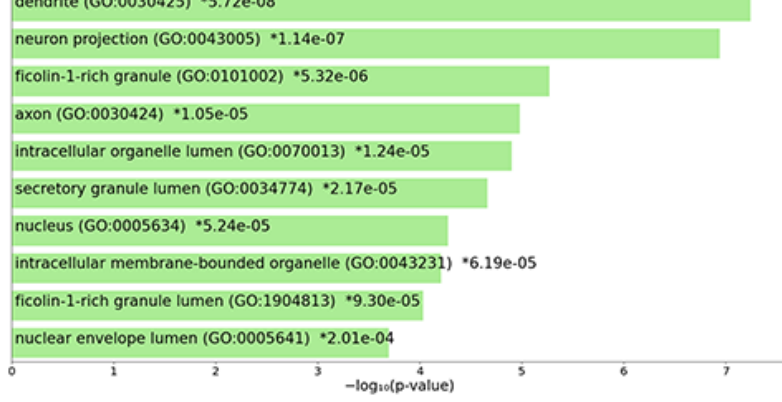

c

transmembrane receptor protein tyrosine kinase signaling pathway (G0:0007169) $* 5.56 \mathrm{e}-15$

positive regulation of phosphorylation (G0:0042327) $* 2.10 \mathrm{e}-14$

cellular response to cytokine stimulus (GO:0071345) *1.15e-13

protein phosphorylation $(60: 0006468) \cdot 1.87 \mathrm{e}-13$

response to lipid (GO:0033993) $\cdot 3.9 \mathrm{e}-13$

positive regulation of transferase activity (GO:0051347) $\cdot 7.01 \mathrm{e}-12$

regulation of inflammatory response $(60: 0050727) * 1.3 \mathrm{e}-11$

regulation of cell population proliferation (GO:0042127) $* 2.72 \mathrm{e}-11$

peptidyl-tyrosine modification (GO:0018212) $\cdot 2.93 \mathrm{e}-11$

regulation of kinase activity (G0:0043549) $* 6.50 \mathrm{e}-11$

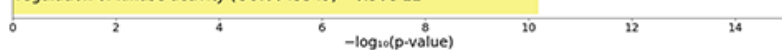

D

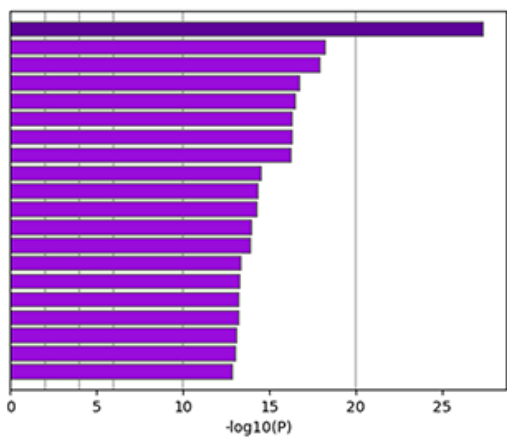

$\mathbf{E}$

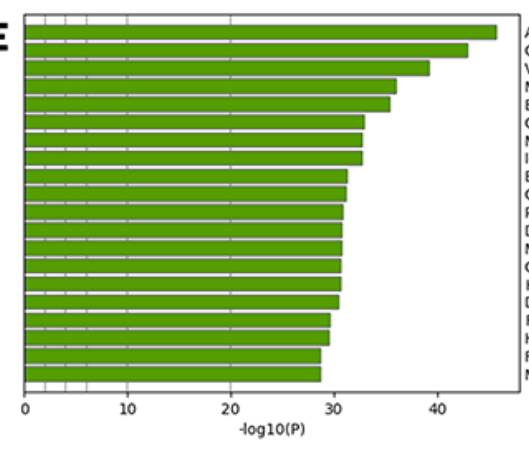

hsa05200: Pathways in cancer GO:0030335: positive regulation of cell migration GO:0010035: response to inorganic substance GO:0071407: cellular response to organic cyclic compound 60:0003013: circulatory system process WP4223: Ras signaling R.HSA-449147: Signaling by Interleukins O.0031667: response to nutrient levels 60:00335: DNA regulation of kinase activity
WP710: DNA dame response (only ATM dependent) GO:1901699: cellular response to nitrogen compound R.HSA-9006931: Signaling by Nuclear Receptors WP2355: Corticotropin-releasing hormone signaling pathway co:0009611: response to wounding GO:0045471: response to ethaxi GO:0000165: MAPK cascade GO:0006091: generation of precursor metabolites and energy
Amyloidosis Cognition Disorders Vascular Diseases Memory impairment Endothelial dysfunction
Cerebral Infarction Middle Cerebral Artery Occlusion diopathic pulmonary arterial hypertension Benign Prostatic Hyperplasia

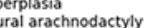
Presenile dementia Mental deterioration Gestational Diabetes Hiabetes Mellitus, Experimental dand Neck Carcinoma Reperfusion Injury
Malignant neoplasm of mouth

\section{Figure 3}

Gene Ontology of 74 genes of common genes of Curcumin targets and AD related targets. Plot representing log-p value and the enriched combination score based on Enrichr. The $p$-value represents the importance of enrichment and the genes/proteins numbers in the group by Enrichr. (A) Molecular function (B) Biological process (C) Cellular component and (D) gene ontology enrichment. (E) Enrichment result by DisGeNet. * Denotes the highly significant ( $p$ value). 


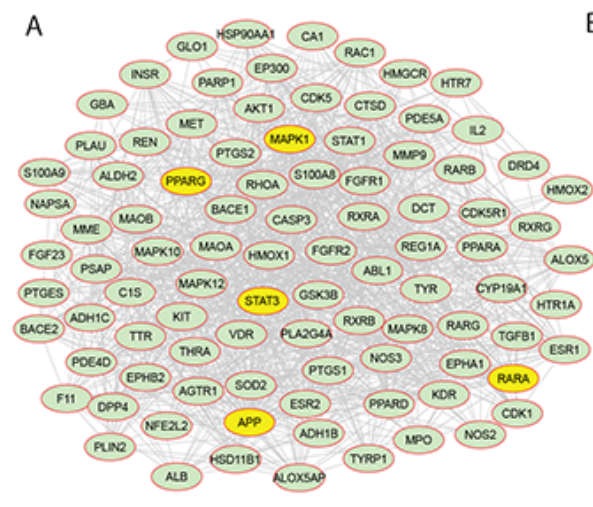

G

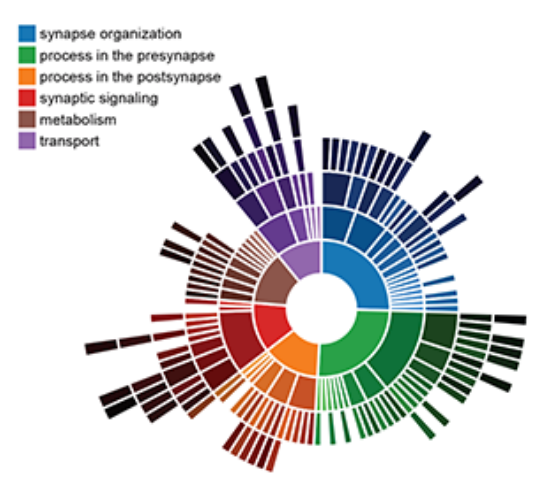

C
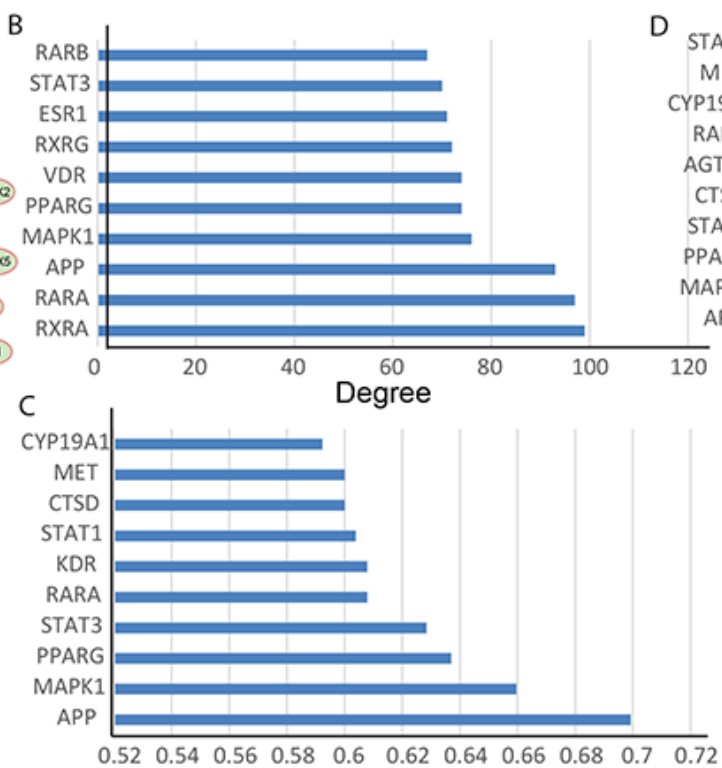

Closeness centrality

$\mathrm{F}$

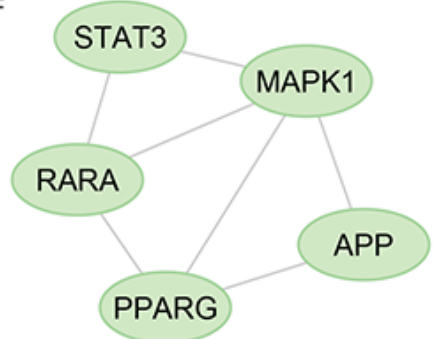

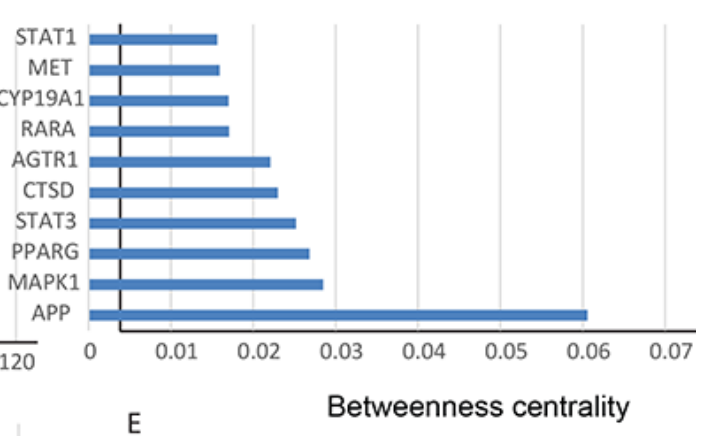

$E$
Betweenness Closeness

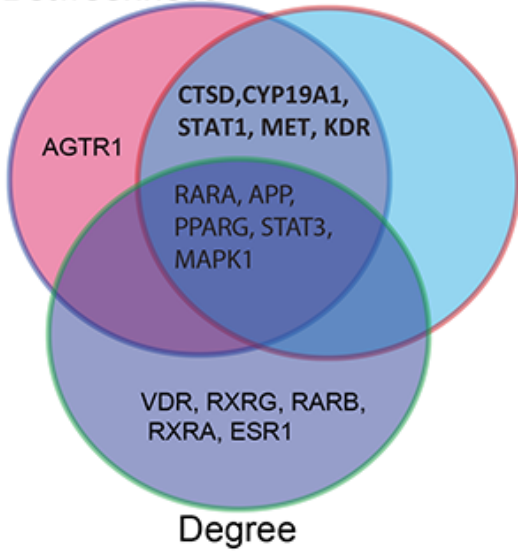

Figure 4

(A) PPI network of gene targeted by curcumin which is associated in AD. (B) The bar graph of top 10 hub gene (C) top 10 ranked genes selected from Betweenness centrality. (D) $\vee$ top 10 ranked genes selected from Closeness centrality. (E) Common gene of top 10 high rank among degree, Betweenness, Closeness centrality. (F) Interaction between the 5 key genes. (G) The significant GO enrichment and pathway enrichment of SynGO genes of 74 common gene among curcumin genes and AD related gene showing biological function of genes in the synapses. 


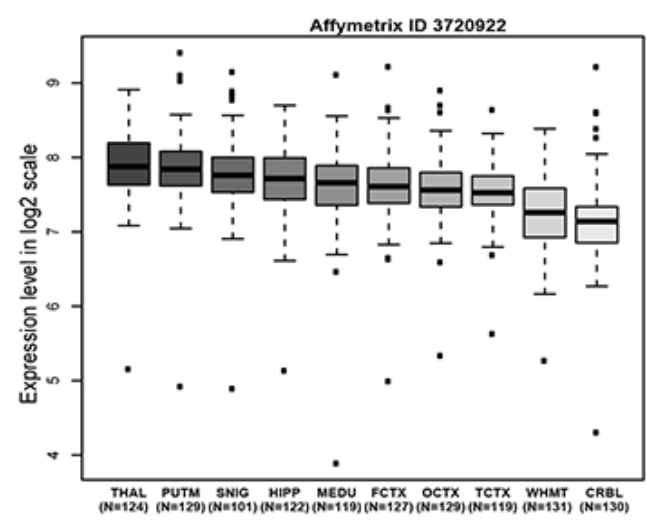

Fold change between THAL and CRBL $=1.7\left(p=2.1 \times 10^{-3 n}\right)$

D. Expression profile of RARA

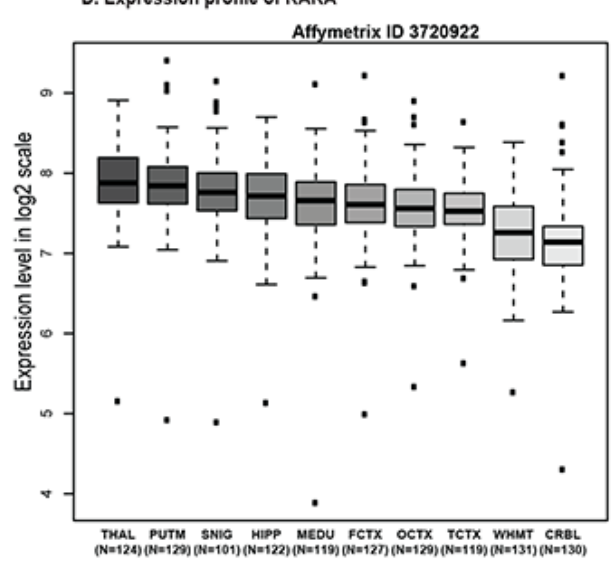

Fold change between THAL and CRBL $=1.7\left(p=2.1 \times 10^{-21}\right)$

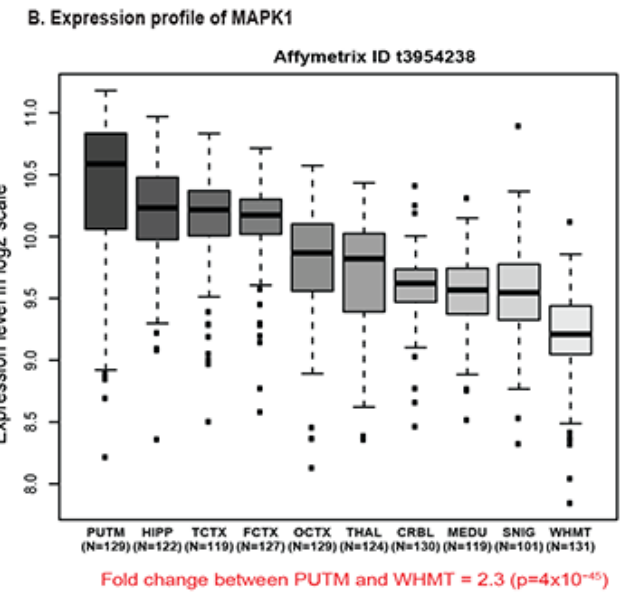

E. Expression profile of APP

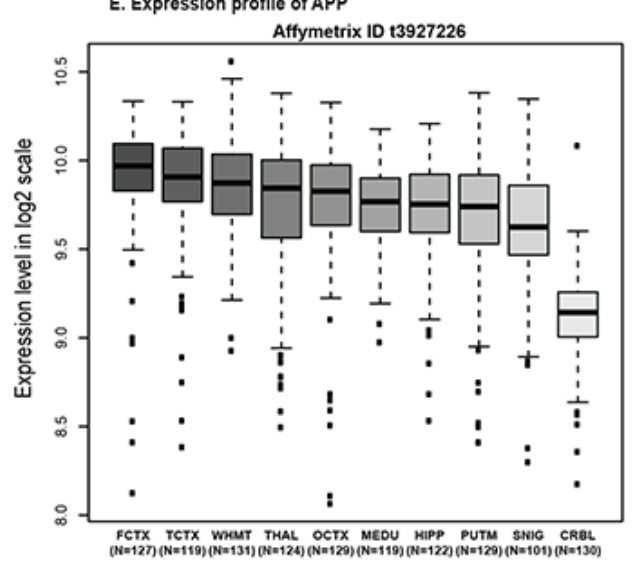

Fold change between FCTX and CRBL $=1.8\left(p=2.6 \times 10^{\circ}\right)$

\section{Expression profile of STAT3}

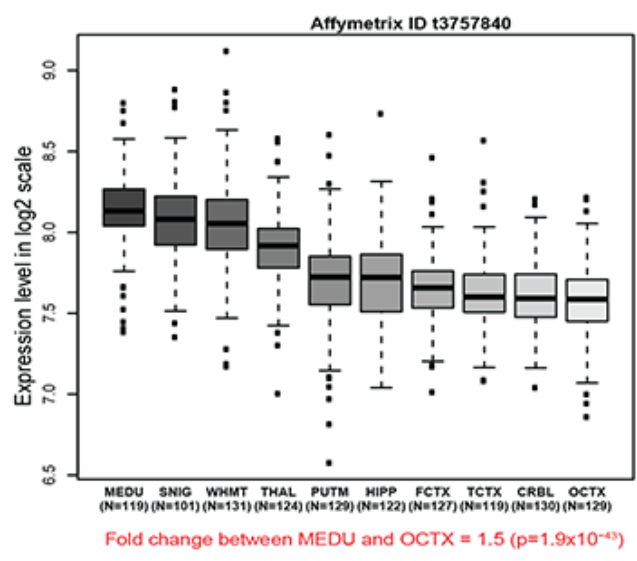

CRBL: cerebellar cortex

FCTX: frontal cortex

OCTX: occipital cortex

TCTX: temporal cortex

HIPP: hippocampus

PUTM: putamen (at the level of the anterior commissure)

THAL: thalamus (at the level of the lateral geniculate nucleus)

MEDU: inferior olivary nucleus (sub-dissected from the medulla)

SNIG: substantia nigra

WHMT: intralobular white matter

\section{Figure 5}

The key gene are expressed in various brain regions. (A) The expression of PPARY. (B) The expression of MAPK1. (C) The expression of STAT3. (D) The expression of RARA. (E) The expression of APP. The transcript level of key gene is retrieved from the BRAINEAC. 
PPARG

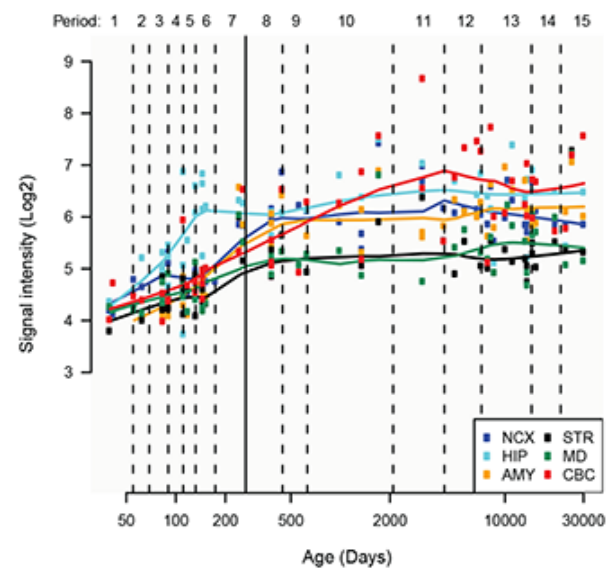

RARA

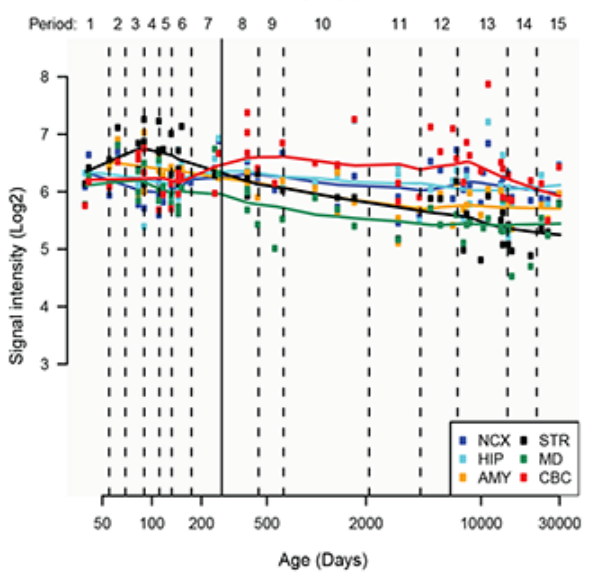

MAPK1
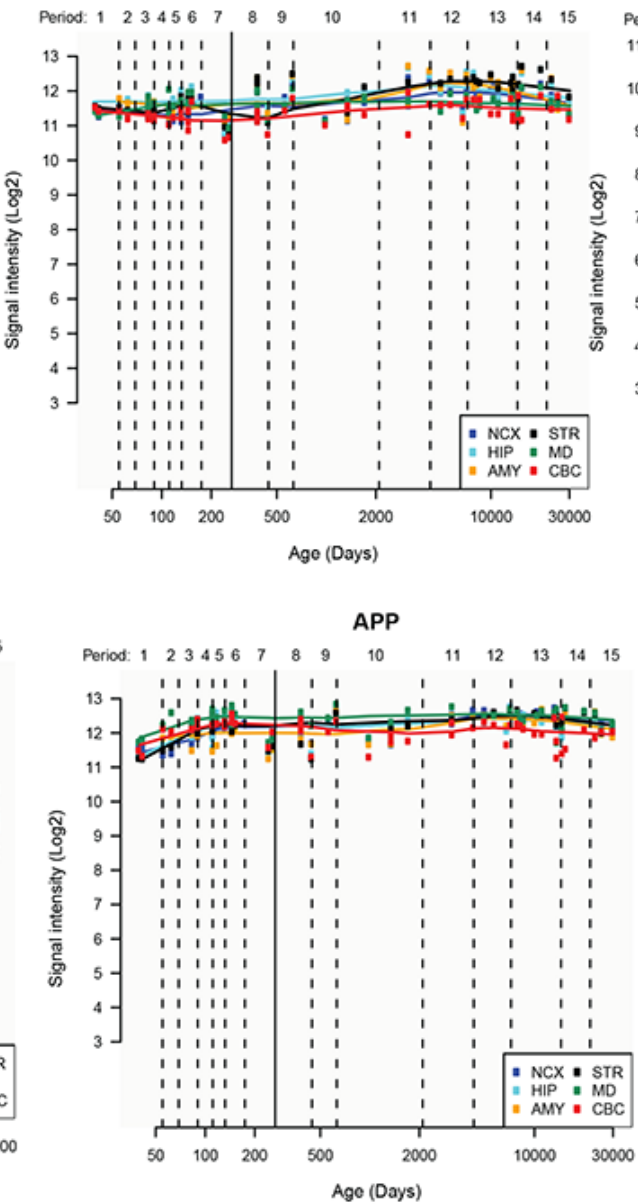

STAT

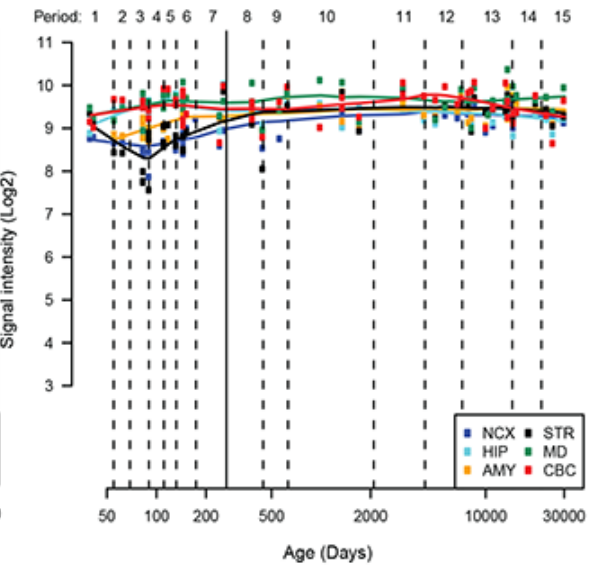

NCX: neocortex HP: hippocampus

AMY: amygdala region

STR: striatum

MD: mediodorsal nucleus of the thalamus CBC: cerebellar cortex.

\section{Figure 6}

Temporospatial expression of PPARy, MAPK1, STAT3, RARA and APP in human CNS tissues. (a) Trajectory plot showing the expression of PPARY, MAPK1, STAT3, RARA and APP during fetal development (period 1-7), infancy (period 8-9), childhood (period 10-11), adolescence (period 12), and adulthood (period 13-15), in individual CNS areas. 

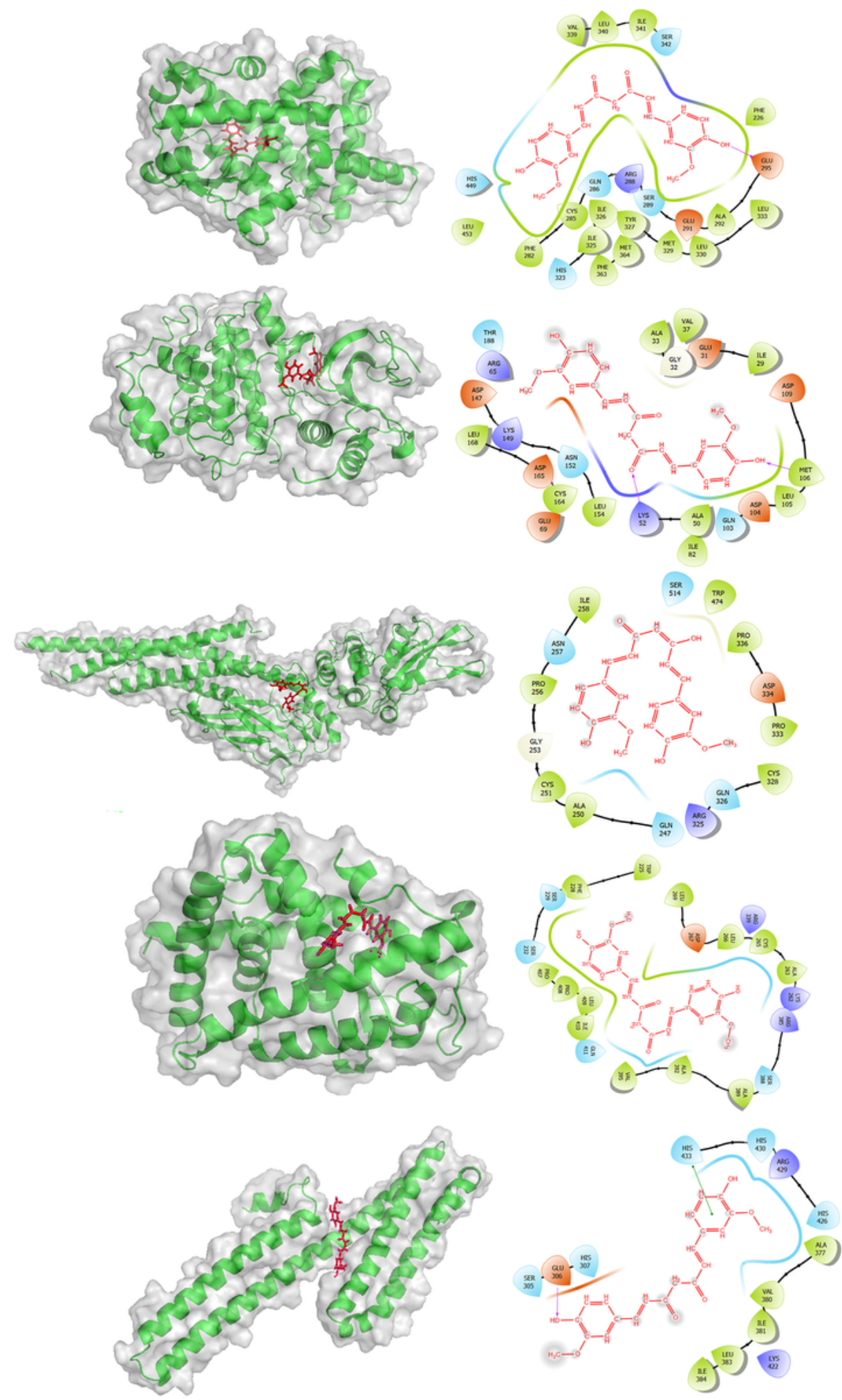

\section{Figure 7}

The docking result of Curcumin and top hub genes using Schrödinger Glide. 3 D and 2 D presentation of interaction between curcumin (A) PPARY (B) MAPK1. (C) STAT3 (D) RARA (E) APP. The red ribbon refers to the protein chain and green refers to the ligand.

\section{Supplementary Files}


This is a list of supplementary files associated with this preprint. Click to download.

- SupplementaryFile1.xlsx 